\title{
Microcarcinoma Papilar da Tiroideia - Relato de Caso: Nódulo Tiroideu Acidental - do Diagnóstico ao Tratamento
}

\section{Thyroid Papillary Microcarcinoma - Case report: Accidental Thyroid Nodule - From Diagnosis to Treatment}

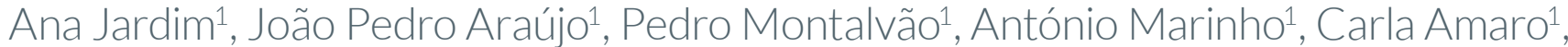
João Cruz², Mónica do Carmo Ferreira² ${ }^{2}$ José Saraiva $^{1}$

\section{RESUMO}

O nódulo tiroideu é um problema clínico muito frequente cuja pertinência clínica é a exclusão de malignidade, que pode representar $4 \%$ a $6,5 \%$ dos casos.

A abordagem diagnóstica do nódulo tiroideu depende de características clínicas e ecográficas que vão determinar os nódulos elegíveis para citologia aspirativa de agulha fina - o resultado (1em 6 possibilidades diagnósticas da classificação de Bethesda) vai ser fundamental na atitude terapêutica.

Este é o relato das etapas de investigação de um nódulo tiroideu detetado acidentalmente, de citologia incerta (lesão folicular de significado indeterminado) que após cirurgia se constatou tratar-se de um microcarcinoma papilar da tiroideia.

PALAVRAS-CHAVE: Carcinoma Papilar/diagnóstico; Carcinoma Papilar/tratamento; Neoplasias da Tiroide; Nódulo da Tiroide/diagnóstico; Nódulo da Tiroide/tratamento

\section{ABSTRACT}

Thyroid nodules are very frequent on clinical daily basis, and ruling out malignancy, which may play the part in up to $4 \%$ a 6.5\% of cases, represents their main clinical interest.

Evaluating a thyroid nodule will depend on clinical as well as ultrasonography characteristics that will further determine which nodules should undergo fine needle aspiration - cytological result (1 out of 6 possibilities according to Bethesda system) will be the main determinant of therapeutic choice.

This is a case report about the evaluation of an incidental thyroid nodule that is cytological indeterminate (follicular lesion of undetermined significance) which proved to be a papillary thyroid microcarcinoma.

KEYWORDS: Carcinoma, Papillary/diagnosis; Carcinoma, Papillary/therapy; Thyroid Neoplasms; Thyroid Nodule/diagnosis;

Thyroid Nodule/therapy 


\section{INTRODUÇÃO}

O nódulo tiroideu é um problema clínico muito frequente, com prevalência entre 1\%-5\% em nódulos cervicais palpáveis, e 16\%-69\% em nódulos detetados em exames complementares, com frequência mais elevada no sexo feminino e em idade avançada.

A pertinência clínica do estudo do nódulo tiroideu é a exclusão de malignidade, uma vez que pode corresponder a cancro da tiroideia em $4 \%$ a 6,5\% dos casos, independentemente de se tratar de um nódulo palpável ou detetado acidentalmente. ${ }^{1-2}$

O carcinoma da tiroideia é a neoplasia endócrina mais comum no mundo, verificando-se uma incidência crescente, refletindo maior disponibilidade e sensibilidade dos meios complementares de diagnóstico.,4

A maioria dos casos (> 90\%) corresponde a carcinoma diferenciado da tiroideia, que inclui os tipos carcinoma papilar e carcinoma folicular. ${ }^{1}$ O carcinoma papilar da tiroideia é sem dúvida o mais frequente (> 80\% dos casos), sendo caracterizado por um excelente prognóstico, sobrevivência global aos 10 anos de cerca de $90 \% .{ }^{5}$ O mesmo prognóstico é esperado para o subtipo microcarcinoma papilar da tiroideia, que de acordo com a Organização Mundial da Saúde (OMS) consiste num carcinoma papilar com dimensão inferior ou igual a $1 \mathrm{~cm},{ }^{6}$ desde que este não apresente características de alto-risco como metástases ganglionares ou à distância, e extensão extratiroideia (nervo laríngeo recorrente, traqueia). ${ }^{7}$

A atitude perante um nódulo tiroideu (vigilância versus investigação adicional) varia de acordo com as características do mesmo. Apenas nos nódulos ecograficamente suspeitos de malignidade e/ou presença de fatores de risco clínico (Tabela 1) está indicada a realização de citologia aspirativa por agulha fina (CAAF) para determinação da classificação citológica. ${ }^{1,8}$

O resultado da citologia deve refletir uma das seis hipóteses diagnósticas da classificação de Bethesda (Tabela 2), e embora não sendo um fator exclusivo, é decisivo na orientação terapêutica (Tabela 3). ${ }^{9}$

Os autores relatam um caso de microcarcinoma papilar da tiroideia diagnosticado em nódulo detetado acidentalmente por ecografia, guiando o leitor desde a avaliação inicial do nódulo tiroideu até ao tratamento e seguimento adotados.

\section{CASO CLÍNICO}

Um homem saudável de 28 anos que recorre à consulta de Otorrinolaringologia (ORL) por pequena tumefação laterocervical esquerda com 2 meses de evolução, assintomática.

Ao exame objetivo destaca-se a presença de adenopatia justacentimétrica na área IIb à esquerda, elástica, móvel, não dolorosa, não aderente aos planos profundos, e sem alterações da pele adjacente. À palpação da tiroideia verifica-se ausência de aumento de volume glandular bem como ausência de formações nodulares. No restante exame objetivo geral e ORL não se detetaram outros achados patológicos.

Foi realizada uma ecografia cervical e tiroideia que demostrou a existência de 2 gânglios na área IIb à esquerda, com 9 mm, inespecíficos; na glândula tiroideia foi detetado no lobo direito um nódulo bem definido, com $13 \times 10$ mm, hipoecogénico, com halo irregular.

Foi ainda realizada uma tomografia computorizada (TC) da faringe e pescoço para esclarecimento das adenopatias - não se detetaram lesões suspeitas nas vias aerodigestivas superiores; os gânglios identificados apresentavam dimensões e morfologia dentro da normalidade; a tiroideia apresentava dimensões normais e contornos

TABELA 1. Fatores de suspeição de malignidade.

\begin{tabular}{|c|c|}
\hline CLÍNICOS & ECOGRÁFICOS \\
\hline $\begin{array}{l}\text { ATA } \\
\text { Antecedentes pessoais, } \\
\text { familiares e sintomas } \\
\text { - Crescimento rápido } \\
\text { de massa cervical } \\
\text { - Disfonia } \\
\text { - Irradiação da cabeça } \\
\text { e pescoço na infância, } \\
\text { irradiação corporal total } \\
\text { para transplante de medula } \\
\text { óssea } \\
\text { - AF cancro da tiroideia } \\
\text { - AF síndromes associadas a } \\
\text { cancro da tiroideia (MEN2, } \\
\text { FAP, S. Cowden) }\end{array}$ & $\begin{array}{l}\text { NCCN } \\
\text { - Nódulo hipoecogénico } \\
\text { - Microcalcificações } \\
\text { - Margens infiltrativas } \\
\text { - Longitudinal > transversal }\end{array}$ \\
\hline $\begin{array}{l}\text { EO } \\
\text { - Paralisia das cordas vocais } \\
\text { - Adenopatias cervicais } \\
\text { - Massa cervical fixa }\end{array}$ & $\begin{array}{l}\text { ATA - Elevada suspeição } \\
\text { (> 70\%-90\%) } \\
\text { Nódulo sólido hipoecogénico ou } \\
\text { componente hipoecogénico em } \\
\text { nódulo misto com um ou + dos } \\
\text { seguintes: } \\
\text { - Margens irregulares } \\
\text { - Microcalcificações } \\
\text { - Longitudinal > transversal } \\
\text { - Halo calcificado com pequenas } \\
\text { extrusões de componente tecidos } \\
\text { moles } \\
\text { - Extensão extratiroideia }\end{array}$ \\
\hline
\end{tabular}

ATA - American Thyroid Association

NCCN - National Comprehensive Cancer Network

MEN2 - multiple endocrine neoplasia type 2

FAP - familial adenomatous polyposis 
TABELA 2. Resultados CAAF - classes diagnósticas recomendadas pelo sistema de BETHESDA.

\begin{tabular}{|c|c|}
\hline I & $\begin{array}{l}\text { Não diagnóstica, insatisfatória } \\
\text { - Líquido de quisto (apenas) } \\
\text { - Espécimen acelular } \\
\text { - Outras (coágulos, artefactos...) }\end{array}$ \\
\hline II & $\begin{array}{l}\text { Benigna } \\
\text { - Consistente com nódulo folicular benigno (nódulo adeno- } \\
\text { matoso, coloide, ...) } \\
\text { - Consistente com tiroidite linfocítica (Hashimoto) } \\
\text { - Consistente com tiroidite granulomatosa (subaguda) }\end{array}$ \\
\hline III & $\begin{array}{l}\text { Atipia de significado indeterminado (ASI) ou lesão folicular } \\
\text { de significado indeterminado (LFSI) }\end{array}$ \\
\hline IV & $\begin{array}{l}\text { Neoplasia folicular ou suspeita de neoplasia folicular } \\
\text { - Especificar se subtipo de Células de Hurthle (oncocítico) }\end{array}$ \\
\hline V & $\begin{array}{l}\text { Suspeita de malignidade } \\
\text { - Suspeita de carcinoma papilar } \\
\text { - Suspeita de carcinoma folicular } \\
\text { - Suspeita de carcinoma metastático } \\
\text { - Suspeita de linfoma }\end{array}$ \\
\hline VI & $\begin{array}{l}\text { Maligna } \\
\text { - Carcinoma papilar da tiroideia } \\
\text { - Carcinoma pouco diferenciado } \\
\text { - Carcinoma medular da tiroideia } \\
\text { - Carcinoma indiferenciado (anaplásico) } \\
\text { - Carcinoma pavimentocelular } \\
\text { - Carcinoma com características mistas (especificar) } \\
\text { - Carcinoma metastático } \\
\text { - Linfoma não-Hodgkin } \\
\text { - Outra }\end{array}$ \\
\hline
\end{tabular}

CAAF - Citologia aspirativa de agulha fina

TABELA 3. Relação entre classe diagnóstica (BETHESDA) - risco de malignidade - atitude.

\begin{tabular}{|l|l|c|c|}
\hline I & \multicolumn{1}{|c|}{ Classe Diagnóstica } & $\begin{array}{c}\text { Risco de } \\
\text { Malignidade }\end{array}$ & Atitude \\
\hline II & $\begin{array}{l}\text { Não diagnóstica, } \\
\text { insatisfatória }\end{array}$ & $1-4 \%$ & Repetir CAAF \\
\hline III & $\begin{array}{l}\text { Atipia de significado } \\
\text { indeterminado (ASI) } \\
\text { ou Lesão folicular } \\
\text { de significado } \\
\text { indeterminado (LFSI) }\end{array}$ & 0-3\% & $\begin{array}{c}\text { Seguimento } \\
\text { clínico }\end{array}$ \\
\hline IV & $\begin{array}{l}\text { Neoplasia folicular ou } \\
\text { suspeita de neoplasia } \\
\text { folicular }\end{array}$ & $15-30 \%$ & Repetir CAAF \\
\hline V & $\begin{array}{l}\text { Suspeita de malignidade } \\
\text { VI }\end{array}$ & Maligna & Lobectomia \\
\hline
\end{tabular}

regulares, referenciando-se apenas nódulo isodenso delimitado por um halo hipodenso, com 9 mm, no lobo direito da glândula. Na avaliação analítica não se detetaram alterações, incluindo TSH (thyroid-stimulating hormone) e T4L (tiroxina livre) compatíveis com eutiroidismo.

Perante estes achados foi pedida uma citologia aspirativa de agulha fina (CAAF) ecoguiada dirigida ao nódulo do lobo direito da tiroideia. A conclusão do exame citológico foi de "lesão folicular de significado indeterminado" (Classificação Bethesda - Tabela 2).

Atendendo à presença de um nódulo tiroideu sólido, solitário, unilateral, intratiroideu, e de citologia incerta, em adulto jovem do sexo masculino, foi colocada indicação cirúrgica de lobectomia direita.

A cirurgia, realizada através da técnica clássica por cervicotomia, decorreu sem intercorrências, com identificação e preservação do nervo laríngeo recorrente direito e das glândulas paratiroideias. No final do procedimento foi colocado dreno aspirativo que drenou conteúdo serohemático vestigial, pelo que foi retirado às 24 horas. Não se verificaram intercorrências pós-operatórias, tendo o doente alta para o domicílio.

O exame anatomopatológico revela na peça de lobectomia direita (dimensões 40 × 35 × 10 mm) a presença de um nódulo com $10 \times 10 \times 8$ mm, sólido, bem circunscrito, revestido por cápsula fina, com consistência firme, sem invasão capsular, linfovascular ou extensão extratiroideia, distando $20 \mathrm{~mm}$ da margem ístmica. Histologicamente este nódulo corresponde a microcarcinoma papilar da glândula tiroideia - variante folicular encapsulado (Fig. 1) - reclassificado como variante folicular não invasiva com características nucleares de tipo papilar.

A estratégia de seguimento adotada após lobectomia, de acordo com as recomendações da NCCN, ${ }^{7}$ foi a vigilância ativa, através de observações periódicas incluindo avaliações clínica, analítica e imagiológica.

\section{DISCUSSÃO}

No decorrer da investigação de uma pequena tumefação laterocervical esquerda num adulto jovem, sexo masculino, saudável, cujo exame objetivo ORL e TC cervical revelaram ausência de significado patológico, foi identificado acidentalmente através de ecografia, um nódulo no lobo direito da tiroideia.

Sabendo que o risco de malignidade é igual para nódulos palpáveis e acidentais (detetados em exames de imagem) é essencial avaliar a presença de fatores de risco clínicos e ecográficos para malignidade (Tabela 1). ${ }^{1}$ 
Apesar da clínica e exame objetivo serem pouco sensíveis para a deteção de cancro da tiroideia, alguns fatores aumentam a probabilidade de malignidade (Tabela 1).

Deverá ser sempre realizada uma ecografia tiroideia para avaliação de critérios ecográficos sugestivos de malignidade (Tabela 1).1,10

A mensuração da TSH sérica é também recomendada em todos os casos, não só para determinação da função tiroideia (eutiroidismo, hipotiroidismo ou hipertiroidismo), mas também com efeito prognóstico - a TSH sérica constitui um fator preditivo independente para o risco de cancro tiroideu, aumentando o risco com o aumento do nível sérico desta hormona. ${ }^{11}$

Em relação à avaliação ecográfica de um nódulo tiroideu, outras características além do seu tamanho (superior ou inferior a $1 \mathrm{~cm}$ ) podem sugerir malignidade. Neste caso há duas características suspeitas, nomeadamente a hipoecogenicidade e as margens irregulares (Tabela 1). Se a dimensão do nódulo fosse $\geq 1 \mathrm{~cm}$, este seria um nódulo com elevada suspeição de malignidade (> 70\%-90\%) e com indicação para CAAF.1,7 Nos nódulos < $1 \mathrm{~cm}$, na ausência de extensão extratiroideia, metástases cervicais ou à distância, e sabendo que os carcinomas micropapilares ( $\leq 1 \mathrm{~cm})$ têm habitualmente um comportamento indolente, a CAAF pode ser dispensada, mantendo-se a vigilância. No entanto, alguns fatores, como a idade, podem mudar o algoritmo de investigação e favorecer a CAAF, uma vez que doentes abaixo dos 40 anos podem ter crescimento tumoral mais agressivo. Neste caso, trata-se de um nódulo sólido justacentimétrico (9-13 mm, dependendo da avaliação por ecografia ou TC), hipoecogénico e de margens irregulares, num adulto jovem, sexo masculino (fator de mau prognóstico), pelo que se optou pela CAAF; pela dimensão do nódulo, optou-se por punção ecoguiada para aumentar a sensibilidade diagnóstica.

O resultado citológico foi compatível com "lesão folicular de significado indeterminado (LFSI)", com risco de malignidade variável entre 5\%-15\% (Tabela 3). Nestes casos, está indicada repetição de CAAF, idealmente ecoguiada e por citopatologista experiente. Resultados semelhantes ou inconclusivos podem motivar estudo molecular ou realização de cirurgia.

O principal objetivo da cirurgia em citologias indeterminadas (como LFSI) é fornecer um diagnóstico histológico definitivo, bem como permitir exérese definitiva da lesão. Num doente com nódulo indeterminado solitário, a lobectomia é a abordagem inicial recomendada. Esta pode ser alterada segundo características clínicas, ecográficas e moleculares ou de acordo com a preferência do doente (Tabela 4).
TABELA 4. Critérios (pré-operatórios ou intraoperatórios) que determinam a cirurgia inicial no carcinoma papilar da tiroideia (NCCN).

\begin{tabular}{|l|l|}
\hline $\begin{array}{l}\text { Indicações para } \\
\text { TIROIDECTOMIA TOTAL }\end{array}$ & Indicações para \\
(se UM presente) & TOMIA (se TODOS presentes) \\
\hline $\begin{array}{l}\text { - Metástases à distância } \\
\text { - Extensão extratiroideia }\end{array}$ & - Sem metástases à distância \\
- Tumor diam. $>4 \mathrm{~cm}$ & - Sem extensão extratiroideia \\
- Metástases ganglionares & - Tumor $\leq 4 \mathrm{~cm}$ \\
cervicais & -Sem metástases ganglionares \\
- Considerar previamente & - Sem irradiação prévia \\
irradiação & \\
- Considerar modularidade & \\
bilateral & \\
- Tumor pouco diferenciado & \\
\hline
\end{tabular}

NCCN - National Comprehensive Cancer Network

TABELA 5. Critérios que determinam atitude após lobectomia ou hemitiroidectomia no carcinoma papilar da tiroideia (NCCN).

\begin{tabular}{l|l} 
Indicações para & Indicações para \\
TOTALIZAÇÃO & SEGUIMENTO* \\
(se UM presente) & (se TODOS presentes) \\
\hline - Margens cirúrgicas positivas & - Margens cirúrgicas negativas \\
- Extensão extratiroideia & - Sem lesão contralateral \\
grosseira & - Diagnóstico histológico de \\
- Tumor > 4 cm & neoplasia folicular tiroideia não \\
- Metástases ganglionares & invasiva com características \\
cervicais macroscópicas & nucleares de tipo papilar \\
- Invasão vascular & (reclassificação: ex-variante \\
& folicular encapsulado do carcinoma \\
& papilar da tiroideia)** \\
& \\
\hline
\end{tabular}

* considerar avaliação da tiroglobulina e dos anticorpos anti-Tg às 6-12 semanas de pós-operatório; considerar terapia com levotiroxina para manter níveis de TSH baixos ou normais.

** nesta variante histológica (reclassificada) apenas a lobectomia é necessária.

NCCN - National Comprehensive Cancer Network
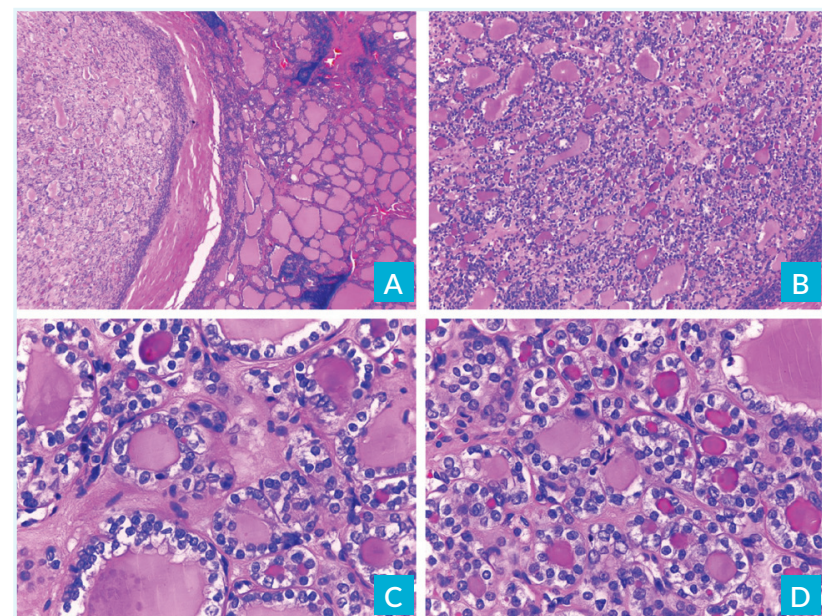

FIGURA 1. Microcarcinoma papilar, variante folicular encapsulado. A - Neoplasia capsulada em contexto de tiroidite linfocítica (HE 40x); B - Neoplasia de padrão folicular (HE 100x); C e D - Núcleos grandes, clarificados, com moldagem, pseudoinclusões intranucleares e fendas longitudinais (HE 400x). 
Assim, neste caso de nódulo sólido justacentimétrico, solitário, intratiroideu e unilateral direito, com citologia indeterminada (LFSI), num adulto jovem do sexo masculino, optou-se pela realização de lobectomia direita, sendo o exame anatomopatológico deste nódulo compatível com microcarcinoma papilar da tiroideia - variante folicular encapsulado - reclassificado como variante folicular não invasiva com características nucleares de tipo papilar. Perante este subtipo histológico, e perante a ausência de critérios para totalização de tiroidectomia (Tabela 5), a atitude adotada foi a vigilância clínica, analítica (tiroglobulina e anticorpos anti-Tg) e imagiológica (ecografia).

CONFLITOS DE INTERESSE: Os autores declaram não ter qualquer conflito de interesse na realização do presente trabalho.

FONTES DE FINANCIAMENTO: Não houve qualquer fonte de financiamento na realização do presente trabalho.

CONFIDENCIALIDADE DOS DADOS: Os autores declaram ter seguido os protocolos da sua instituição acerca da publicação dos dados de doentes.

PROTEÇÃO DE PESSOAS E ANIMAIS: Os autores declaram que os procedimentos seguidos na elaboração do presente trabalho estão em conformidade com as normas das comissões de investigação clínica e de ética, bem como da declaração de Helsínquia e da Associação Médica Mundial.

CONFLICTS OF INTEREST: The authors declare that they have no conflicts of interest.

FINANCIAL SUPPORT: This work has not received any contribution, grant or scholarship.

CONFIDENTIALITY OF DATA: The authors declare that they have followed the protocols of their work center on the publication of data from patients.

PROTECTION OF HUMAN AND ANIMAL SUBJECTS: The authors declare that the procedures followed were in accordance with the regulations of the relevant clinical research ethics committee and with those of the Code of Ethics of the World Medical Association (Declaration of Helsinki).

\section{REFERÊNCIAS}

1. Haugen BR, Alexander EK, Bible KC, Doherty GM, Mandel SJ, Nikiforov YE, et al. 2015 American Thyroid Association management guidelines for adult patients with thyroid nodules and differentiated thyroid cancer: The American Thyroid Association Guidelines Task Force on Thyroid Nodules and Differentiated Thyroid Cancer. Thyroid 2016;26:1-133.

2. Lin JD, Chao TC, Huang BY, Chen ST, Chang HY, Hsueh C. Thyroid cancer in thyroid nodules evaluated by ultrasonography and fine-needle aspiration cytology. Thyroid 2005;15:70817.

3. Vinciguerra GLR, Noccioli N, Bartolazzi A. Diffuse follicular variant of papillary thyroid carcinoma: a case report with a revision of literature. Rare Tumors. 2016;8:6536.

4. Wang T, Xu H, Qi M, Yan S, Tian X. miRNA dysregulation and the risk of metastasis and invasion in papillary thyroid cancer: a systematic review and meta-analysis. Oncotarget. 2017; 9:5473-9.

5. Shin JH. Ultrasonographic imaging of papillary thyroid carcinoma variants. Ultrasonography. 2017;36:103-10.

6. World Health Organization. Classificiation of Tumours: Pathology and Genetics of Tumours of the Endocrine Organs. 3rd ed. Lyon: IARC Press; 2004.

7. Ito Y, Miyauchi A, Oda H. Low-risk papillary microcarcinoma of the thyroid: A review of active surveillance trials, Eur J Surg Oncol. 2018;44:307-15.

8. National Comprehensive Cancer Network. NCCN Guidelines for Thyroid Carcinoma. Version 1.2017- March 31, 2017. Bethesda: NCCN; 2017.

9. Cibas ES, Ali SZ. The Bethesda system for reporting thyroid cytopathology. Am J Clin Pathol. 2009;132:658-65.

10. US Preventive Services Task Force, Bibbings-Domingo K, Grossman DC, Curry SJ, Barry MJ, Davidson KW, et al. Screening for thyroid cancer: US Preventive Task Force Recommendation Statement. JAMA 2017;317:1882-7.

11. Boelaert K, Horacek J, Holder RL, Watkinson JC, Sheppard MC, Franklyn JA. Serum thyrotropin concentration as a novel predictor of malignancy in thyroid nodules investigated by fine-needle aspiration. J Clin Endocrinol Metab. 2006:91:4295301. 